\title{
La Chine au Kenya : enjeu stratégique dans un pays pauvre en ressources naturelles
}

David Bénazéraf

\section{Q OpenEdition \\ 1 Journals}

\section{Édition électronique}

URL : https://journals.openedition.org/eastafrica/338

DOI : 10.4000/eastafrica.338

ISSN : 2790-1076

Éditeur

IFRA - Institut Français de Recherche en Afrique

Édition imprimée

Date de publication : 1 septembre 2014

Pagination : 7-27

ISSN : 2071-7245

\section{Référence électronique}

David Bénazéraf, «La Chine au Kenya : enjeu stratégique dans un pays pauvre en ressources

naturelles », Les Cahiers d'Afrique de l'Est / The East African Review [En ligne], 49 | 2014, mis en ligne le 07 mai 2019, consulté le 09 décembre 2021. URL : http://journals.openedition.org/eastafrica/338 ; DOI : https://doi.org/10.4000/eastafrica.338 


\title{
La Chine au Kenya : enjeu stratégique dans un pays pauvre en ressources naturelles
}

\author{
David Bénazéraf
}

\begin{abstract}
Résumé : La Chine est devenue en quelques années un partenaire majeur du Kenya. Première source des investissements directs à l'étranger entrants au Kenya et deuxième partenaire commercial, elle est aussi son premier bailleur de fond bilatéral. Sans être un fournisseur majeur de ressources naturelles, le Kenya constitue toutefois un pays clé pour la stratégie chinoise en Afrique de l'Est. Les échanges économiques sino-kenyans ne représentent qu'un volet de la présence chinoise dans le pays. La Chine tente également d'y accroitre son influence culturelle et son pouvoir d'attraction. Elle reçoit un accueil favorable des autorités dans le cadre de la politique d'ouverture aux pays asiatiques.
\end{abstract}

\section{Introduction}

Bien que l'Afrique de l'Est soit encore peu pourvue en richesses naturelles de grande ampleur identifiées et exploitées, la Chine entretient des relations économiques importantes avec les acteurs des pays de la région (Éthiopie, Tanzanie et Kenya principalement). Ces relations couvrent de nombreux secteurs, comme c'est le cas sur l'ensemble du continent africain. La présence des acteurs chinois, ancienne pour les entreprises publiques et plus récente pour les entreprises privées, est en effet en forte croissance sur l'ensemble du continent africain depuis les années 2000, et dans des secteurs très variés. Les relations de l'État chinois avec ses partenaires africains ont été renforcées depuis le premier Forum sur la coopération sino-africaine qui s'est tenu en 2000 (Kernen, 2007 ; Taylor, 2011) et à travers les liens que développent les provinces chinoises avec les États africains. La Chine occupe désormais une place de premier plan dans de nombreux pays d'Afrique. La consolidation de sa présence était régulièrement décrite comme une manière de sécuriser ses approvisionnements en matières premières pour alimenter la demande intérieure. Les ressources naturelles sont considérées comme structurantes dans les relations sinoafricaines (Alden \& Soares, 2008). La structure des échanges commerciaux demeure ainsi fondamentalement de type Nord-Sud : exportation de ressources naturelles ou

1 Doctorant en géographie, Université Paris I Panthéon-Sorbonne, Laboratoire Prodig CNRS-IRD. 
agricoles par les pays africains qui importent des biens manufacturés, limitant d'autant le développement d'une industrie locale et favorisant les phénomènes de rente (Chaponnière, 2012). L'accélération des relations Chine-Afrique exacerbe la confrontation entre les bailleurs à l'échelle globale (Tan, 2010). La Chine est qualifiée d'acteur extérieur, émergent, venant s'immiscer dans les relations entre anciens colonisés et anciens empires coloniaux ( $\mathrm{Li}, 2006)$ en dépit de sa politique de coopération pourtant ancienne sur le continent (Chaponnière, 2008). Le concept de "Chinafrique'2 perpétue un certain nombre de mythes sur l'importation massive d'une main-d'œuvre chinoise bon marché par exemple. Pourtant, les modalités de la présence chinoise sont diverses et font appel à différents outils et stratégies (aide, commerce, investissements) en fonction des contextes particuliers de chacun des pays (Brautigam, 2009). Les Chinois ne sont pas seulement acheteurs de matières premières ; ils sont de plus en plus en position d'investisseurs (Veron, 2008). En dépit de constantes, leurs interventions varient en fonction des 49 pays africains avec lesquels la Chine entretient des relations diplomatiques.

En-deçà de l'approche générale sur les relations Chine-Afrique, des nuancess'imposent au regard des relations particulières qu'entretiennent l'État chinois et l'ensemble de ses acteurs, publics ou privés, avec chacun des acteurs des pays africains. Nous analyserons dans cet article les spécificités des relations sino-kenyanes en examinant l'ensemble des volets concernés, à l'exception de la coopération militaire ${ }^{3}$. Si le contenu de ces relations a été passé en revue ces dernières années (Onjala, 2008 ; Mutua Kioko, 2012), la présence chinoise peut être replacée dans le contexte plus large de son engagement en Afrique, et analysée au regard de la diplomatie d'influence de la Chine. La Chine est en effet devenue le premier bailleur bilatéral du Kenya en 2011 et constitue depuis 2013 la première source d'investissement direct à l'étranger (Ambassade de Chine au Kenya). Le Kenya est luimême le troisième partenaire de la Chine parmi les 24 pays d'Afrique australe et de l'Est. En l'espace d'une décennie, la Chine s'est imposée comme un acteur majeur dans le pays, concurrençant les partenaires et bailleurs historiques du Kenya. Les acteurs chinois sont toutefois en compétition avec d'autres acteurs asiatiques ou émergents, comme le

2 Ce néologisme faisant référence à la 'Françafrique' ouvre la voie à une lecture post-coloniale et essentialiste des relations entre la Chine et l'Afrique. Il convient de se départir du contexte historique de la colonisation, et de considérer 'la Chine' non pas comme un bloc monolithique, mais comme un ensemble d'acteurs pluriels. 3 La première délégation militaire chinoise se rend au Kenya en décembre 1996, dirigée par le commandeur de la région militaire de Lanzhou. D'autres visites peuvent être relevées comme celle du général Li Jinai, commissaire politique de la direction des équipements de l'armée chinoise en octobre 2000, puis en décembre 2001, celle du général Fu Quanyou, chef de département des ressources humaines. Le Kenya dispose en outre d'un attaché militaire en Chine depuis 1998 (Mutua Kioko, 2012 : 12). 
Japon, la Corée ou encore Singapour souvent cité en modèle (Xinhua, 2010). En tant que centre économique de la région avec une situation politique globalement stable, le Kenya constitue une base-arrière pour les acteurs économiques chinois en Afrique de l'Est, comme c'est déjà le cas pour d'autres acteurs internationaux. Les deux pays entretiennent des relations dans des domaines diversifiés : agriculture, santé, tourisme, médias, énergie, infrastructures, etc.

Cet article combine deux approches de la géographie : les études sur le développement et la géopolitique. Au sein du cadre général des études sur les relations ChineAfrique, il mêle une approche macro à l'échelle interétatique (entre deux États, la Chine et le Kenya, et non pas entre la Chine et le continent africain pris dans son ensemble), et une approche micro à travers l'étude des acteurs supports de ces relations (Monson \& Rupp, 2013). Le propos est basé sur des sources bibliographiques et sur une enquête de terrain. Par ailleurs, nous distinguons la Chine en tant qu'État et la pluralité des acteurs en provenance de ce pays.

En quoi le Kenya constitue-t-il, malgré l'absence de ressources naturelles majeures, un point d'entrée stratégique pour la Chine en Afrique de l'Est? Comment les relations sino-kenyanes ont-elles évolués ? Quels sont les différents acteurs et vecteurs de la présence chinoise au Kenya ? Après avoir dressé un état des lieux des relations sino-kenyanes et de la présence chinoise dans le pays, nous verrons en quoi les relations commerciales, de coopération, et d'investissements sont caractérisées par une diversité importante. Enfin, nous analyserons dans quelle mesure le Kenya constitue un point d'entrée du soft power ${ }^{4}$ chinois en Afrique.

\section{Une intensification progressive des relations sino-kenyanes}

\section{Des contacts anciens}

Les premiers contacts des Chinois avec l'Afrique ont eu lieu sous la dynastie des Ming pendant une courte phase d'ouverture au XVème siècle, sur les côtes de l'actuel Kenya. Alors que l'histoire chinoise est marquée par une succession de périodes d'ouverture et de repli, le troisième empereur de la dynastie des Ming, Zhu Di (14021424), commandite plusieurs expéditions vers l'océan indien à partir de 1405. A la tête de 200 navires et 27000 hommes, l'amiral Zheng He mènera sept expéditions pacifiques

4 Le soft power sera ici entendu comme un ensemble d'outils non coercitifs rendant possible une capacité d'attraction en terme d'idées, de culture ou de valeurs (Nye, 2004). 
vers les Indes, l'Arabie, et jusqu'aux côtes d'Afrique orientale. Les navires transportent des soldats mais aussi des traducteurs et des savants. Toutefois, l'Empire chinois ne tente pas d'annexion loin de sa sphère d'influence. Les guerres dans le Nord de la Chine mettent fin aux expéditions et l'ouverture maritime se termine dès 1433 (Bezbakh, 2011), mais les relations se poursuivent entre l'Asie et les côtes d'Afrique de l'Est par le biais des routes commerciales terrestres (ivoire, pierres précieuses) pendant plusieurs siècles. À la fin du XIXème siècle, les Britanniques font venir des ouvriers indiens et chinois pour la construction du chemin de fer reliant la côte kenyane à l'actuel Ouganda. Contrairement à certains ouvriers indiens qui s'établissent dans le pays, les Chinois ne restent pas après les travaux. C'est d'ailleurs encore le cas aujourd'hui : la plupart des ouvriers ou ingénieurs chinois travaillant sur des chantiers en Afrique retournent en Chine après une période d'une à trois années 5 .

Des relations diplomatiques qui prennent leur essor à partir de 1978

Les relations entre la Chine et le Kenya sont officialisées quelques jours après l'indépendance du pays, avec l'établissement des relations le 14 décembre 1963. Un premier accord commercial est signé dès 1964 mais les relations diplomatiques demeurent au niveau chargé d'affaires (sans ambassadeur) jusqu'aux années 1970. Celles-ci ne connaissent un réel essor qu'à partir de l'accession à la présidence de Daniel Arap Moi en 1978. Cette année marque également le début des réformes d'ouverture de Deng Xiaoping en Chine après l'ère maoïste : l'idéologie n'est plus alors le seul déterminant des relations internationales de la Chine. En 1978, les deux pays signent un accord de coopération économique et technologique, un nouvel accord commercial, ainsi que plusieurs contrats de construction, puis un accord de coopération culturelle en 1980 (Onjala, $2008: 5$ ). La Chine construit notamment le stade de Kasarani à Nairobi (60 000 places) en 1986 pour les Jeux panafricains, conduisant ainsi une « diplomatie des stades» (Will, $2012: 151$ ).

L'ère du président Arap Moi (1978-2002) est marquée par des visites mutuelles régulières. Arap Moi se rend en Chine trois fois, en septembre 1980, en octobre 1988, et en mai 1994. Le Kenya est représenté par son Ministre des Affaires étrangères, Bonaya Adhi Godana, pour le premier Forum sur la coopération sino-africaine (Focac) en 2000. Entre 1978 et 2002, le Kenya accueille près de 20 visites chinoises d'importance, dont le Premier Ministre Zhao Ziyang en janvier 1983, le Président Jiang Zemin en mai

5 Entretiens avec des salariés d'entreprises de construction chinoises en Chine, au Kenya et en Angola. 
1996 et le Premier Ministre Zhu Rongji en avril 2002, ainsi que trois visites de ministres chinois des Affaires étrangères en 1991, 1994 et 1999. Un accord sur l'éducation supérieure est signé en 1994 ainsi qu'un accord sur la promotion et la protection des investissements en 2001.

Après l'accession à la présidence de Mwai Kibaki (2002-2013), Le Kenya accorde une importance croissante à la Chine. Un nouvel élan est donné à partir de 2002. La coalition gouvernementale au pouvoir instaure alors une stratégie politique d'ouverture envers les pays asiatiques, dite Look East Policy ${ }^{6}$. Le Kenya se tourne vers d'autres modèles de développement pour ne plus se limiter au seul modèle occidental. Ce positionnement part du principe selon lequel l'Asie, par son développement récent, peut inspirer le développement du Kenya, à un moindre coût par rapport aux pays occidentaux grâce à des prêts moins couteux : « the East has also something to offer » ${ }^{7}$, indique le Professeur Peter Kamau de l'Université Kenyatta. Mais on peut y voir également des raisons plus politiques : les pays non-membre de l'OCDE constituent une alternative aux conditions de bonne gouvernance imposées par les pays occidentaux.

Pendant la présidence de Mwai Kibaki, le rythme des échanges diplomatiques s'intensifie, avec trois visites du Président kenyan en Chine en 2005, 2006 pour le troisième Focac, puis en 2010 pour l'inauguration de l'Exposition universelle de Shanghai. Plus de 15 ministres kenyans se sont rendus en Chine depuis 2002 (Ministres des Affaires étrangères, des Finances, des Communications, des Routes et travaux publics, du Tourisme). Côté chinois, plusieurs visites de hauts responsables à Nairobi témoignent de la place accordée au Kenya par la diplomatie chinoise, comme celles de Wu Banguo, Président de l'Assemblée nationale populaire, en 2004, de Jia Qinglin, Président de l'Assemblée consultative, en 2007, ou encore de Li Zhangchun, membre du bureau politique du comité permanent du Parti communiste, en 2011 (Ministère chinois des Affaires étrangères). Le Président $\mathrm{Hu}$ Jintao effectue une étape au Kenya lors de sa tournée africaine en avril 2006. Cette visite est marquée par la signature de nombreux contrats, notamment l'octroi d'un prêt pour la construction d'infrastructures et de projets dans le secteur de l'énergie, d'une subvention de 60 millions RMB pour la coopération technique, d'une subvention de 5 millions RMB pour la fourniture par la Chine de traitements contre la malaria, pour le développement des liaisons aériennes entre les deux pays, pour une assistance technique

6 « Politique regarder vers l'Est. »

7 « L'Orient a aussi des choses à offrir. » 
sur les normes industrielles, et pour la modernisation des équipements de la Kenya Broadcasting corporation (Onjala, 2008 : 7 ; Mutua Kioko, 2012 : 6). Plus récemment encore, le Président Uhuru Kenyatta, élu en avril 2013, s'est rendu en août 2013 à Pékin où il cherche en la Chine un allié de taille. Un prêt de cinq milliards US dollars ${ }^{8}$ a été signé. Il financera notamment l'amélioration des infrastructures dans le cadre de la Vision 2030 : construction d'une ligne de chemin de fer vers l'Ouganda entre Mombassa et Malaba selon les normes ferroviaires chinoises, projets dans le domaine de l'énergie ainsi que la protection de la faune sauvage ${ }^{9}$ (Reuters, 2013; Xinhua, 2013). Peu de précisions ont été apportées sur les modalités du prêt ${ }^{10}$. Un projet de création d'une chambre de compensation (clearing house) pour le Yuan à Nairobi a également été annoncé. À moyen terme, Pékin pourrait aussi financer le projet de chemin de fer entre le port de Lamu, le Sud-Soudan et l'Éthiopie dans le cadre du projet LAPSSET ${ }^{11}$ (Amelot, $2011: 187-188$ ).

\section{L'héritage de la guerre froide}

Depuis 1978, les échanges entre la Chine et le Kenya demeurent soutenus, notamment à travers la fréquence et le niveau élevé des visites mutuelles. Le début des relations est toutefois marqué par l'absence d'affinités politiques particulières, contrairement aux relations de la Chine avec les deux autres grands pays d'Afrique de l'Est, l'Éthiopie et la Tanzanie. En effet, la coopération économique sino-éthiopienne est favorisée par des conceptions politiques communes entre les deux pays (Cabestan, 2012 : $57 \mathrm{sq}$.). Le président tanzanien Julius Nyerere (1964-1985) entretient des liens étroits avec la Chine où il se rend 13 fois durant son règne. Dominique Decherf, ancien ambassadeur de France dans plusieurs pays africains, rappelle dans son livre les affinités occidentales de Kenyatta, et les enjeux liés aux influences respectives des sphères occidentales et communistes pendant la guerre froide : « La CIA s'en moquait [du Président Nyerere qui expulse plusieurs milliers d'Indiens de Tanzanie en 1972]. Mais beaucoup moins du fait que le dictateur 'était en train de céder Zanzibar aux Chinois... Car l'île de Zanzibar, véritable porte-avions ancré à vingt kilomètres de la côte tanzanienne, pouvait devenir un Cuba chinois. » (Decherf, $2012:$ 72).

8 Tous les montants financiers sont libellés en US dollars dans cet article.

9 Le volet biodiversité fait suite au trafic avec la Chine de défenses d'éléphants et de rhinocéros braconnés.

10 Celui-ci étant libellé en dollars, il s'agit certainement d'un prêt en argent, les dons en nature étant libellés en Yuan (Brautigam, 2009 : 125).

11 Lamu Port-South Sudan-Ethiopia. 
Le Président Jomo Kenyatta demeure quant à lui proche du bloc occidental. Celui-ci a en effet passé 16 ans à Londres entre 1929 et 1945 et y a conservé ses alliances politiques (Decherf, 2012 : 70-72). Le Kenya ne fait par exemple pas partie de la dizaine de pays visités par Zhou Enlai lors de sa tournée africaine en décembre 1963 et février 1964 (Brautigam, 2009, 32). Le contexte des répercussions de la guerre froide sur le continent africain peut ainsi expliquer pourquoi les relations sino-kenyanes ne se développement véritablement qu'à partir de 1978. Ce lent démarrage n'empêchera pas la Chine et ses acteurs de prendre attache au Kenya.

\section{État des lieux de la présence chinoise au Kenya}

Le Kenya occupe aujourd'hui une place importante dans la politique africaine de la Chine. Nairobi accueillerait une des plus importantes ambassades chinoises sur le continent africain (Chaponnière, 2012 : 146), avec une trentaine de diplomates à la chancellerie et six au sein du Bureau du Conseiller pour la coopération économique et commerciale $^{12}$. Sur le plan des échanges économiques, plusieurs outils et mécanismes contribuent à la facilitation des affaires entre acteurs chinois et kenyans, notamment la chambre de commerce sino-kenyane, basée à Nairobi, sous la tutelle de l'Ambassade de Chine et présidée par un Chinois, M. Gao. Un comité sur les relations économiques et commerciales sino-kenyanes a été mis en place ${ }^{13}$. Un portail Internet dédié à la coopération économique entre les deux pays a également été créé en 2006 à l'initiative du Ministère chinois du Commerce (Mofcom).

Alors que le Kenya comptait très peu de Chinois sur son territoire avant 2002, on en dénombre aujourd'hui entre 8000 et $10000^{14}$. Il existerait même à Nairobi une communauté chrétienne chinoise regroupant environ 300 membres $^{15}$. Le nombre de touristes chinois dans le pays est passé de 20000 en 2008 à 40000 en 2012, le Kenya ayant été désigné comme destination touristique autorisée par l'Administration chinoise du tourisme en 2004.

12 Entretien auprès du Bureau du Conseiller pour la coopération économique et commerciale de l'Ambassade de Chine à Nairobi.

13 La troisième réunion de ce comité s'est tenue le 25 avril 2006 lors de la visite du Président Hu Jintao à Nairobi.

14 Une citoyenne chinoise figure d'ailleurs sur la liste des victimes de l'attaque du Westgate Mall de Nairobi à l'automne 2013.

15 Entretien avec un entrepreneur chinois de Nairobi. 
Sur le plan des acteurs économiques, l'Ambassade de Chine à Nairobi recensait 67 entreprises à capitaux chinois au Kenya fin 2012, tout type d'entreprises confodues (publiques, paraétatiques et privées) ${ }^{16}$. La répartition par secteurs témoigne d'une diversité importante même si le domaine de la construction demeure prépondérant : construction - BTP (25 entreprises), commerce - import-export (11), immobilier ${ }^{17}$ (9), industrie (8), tourisme (5), communications et réseaux (4), secteur bancaire (2), santé (2), ressources naturelles (1). Une seule entreprise classée dans le secteur de l'exploitation des ressources naturelles figure dans la liste ${ }^{18}$. La présence économique chinoise au Kenya ne semble donc pas motivée directement par l'extraction des ressources naturelles, bien qu'elle puisse tirer bénéfice de la part accrue de ce secteur dans l'économie kenyane par l'intermédiaire de son engagement dans d'autres secteurs économiques d'intervention pouvant être associés au secteur des ressources naturelles, comme le bâtiment, l'industrie ou l'importexport. Les entreprises chinoises de construction occupent la première place. Il s'agit principalement de sociétés publiques de rang national ou provincial. Contrairement à de nombreuses entreprises locales, la plupart sont classées 'A' dans la typologie kenyane : elles ont la capacité d'honorer des contrats supérieurs à cinq milliards de shillings kenyans (environ 58 millions dollars). Parmi les 25 entreprises répertoriées comme entreprises de construction, dix d'entre elles figurent dans le classement 2013 des 250 premières entreprises mondiales de construction (Engineering News record).

Si les relations sino-kenyanes sont ralenties durant les premières années qui suivent l'indépendance, elles se renforcent progressivement avec un premier essor à partir de 1978 après l'accession d'Arap Moi pouvoir. Elles connaissent un second élan à partir de 2002 : les années 2000 sont à la fois marquées par le renforcement massif de la présence chinoise en Afrique avec la création du Focac, et par la consolidation des coopérations

16 D'autres sources donnent le chiffre de 50 entreprises (Chaponnière, 2012), voire de 200 (site du Ministère chinois des Affaires étrangères). Nous nous baserons ici sur la liste fournie par le bureau du Conseiller de coopération économique et commerciale de l'Ambassade de Chine au Kenya. Celle-ci ne tient pas cependant compte des petits entrepreneurs, commerçants, propriétaires de restaurants. Ces derniers échappent aux statistiques. Ils sont en outre relativement moins nombreux au Kenya que dans d'autres pays africains, le commerce étant largement tenu par la bourgeoisie locale. En outre, toues les entreprises ne bénéficient pas d'un soutien de l'État chinois (Kernen, $2007: 178$ ).

17 Certaines entreprises appartenant à la catégorie 'immobilier' cumulent les fonctions de promoteur et de constructeur.

18 Il s'agit de la China National Offshore Oil Corporation (CNOOC) qui s'est vue attribuer un contrat d'exploration pétrolière sur un total de six blocs offshore et le long des frontières avec le Sud-Soudan et la Somalie, soit $45500 \mathrm{~km}^{2}$. La CNOOC avait décroché ce contrat d'exploration face à des concurrents européens (l'Espagnol Cepsa et le Suédois Lundin) en 2004 dans le cadre de la politique « Look East » du gouvernement, mais s'est retirée en 2010 sans être parvenue à une exploitation viable commercialement. 
asiatiques du Kenya dans le cadre de la politique Look East. La présence chinoise au Kenya est, depuis cette période, désormais bien établie, et fondée sur des relations diversifiées.

\section{Des relations diversifiées}

Les échanges entre la Chine et le Kenya témoignent d'une pluralité en termes de secteurs économiques d'intervention, ainsi que de modalités de coopération réparties entre aide au développement, commerce et investissements.

\section{Aide au développement}

L'aide chinoise est délivrée par le biais de plusieurs outils : prêts concessionnels, crédits préférentiels acheteurs à l'export, subventions, assistance technique, dons en nature (machines-outils, denrées alimentaires). Elle couvre plusieurs secteurs : construction d'infrastructures, santé, agriculture, énergie. Mais la Chine n'utilise pas les mêmes critères de définition que les pays membres du comité d'aide au développement de l'OCDE et utilise une conception moins restrictive de l'aide à l'étranger. Les comparaisons sont donc rendues difficiles. Il s'agit en outre toujours d'aide liée, c'est-à-dire assujettie à l'achat de biens chinois ou au recours à des entreprises chinoises (Brautigam, 2009 : 152). Celle-ci est délivrée pour des projets déterminés et non comme appui à des politiques sectorielles : il s'agit d'aide projet et non d'aide programme (Charnoz \& Severino 2007). En outre, la coopération sino-africaine, de type sud-sud, est le fruit d'articulations complexes au sein d'un faisceau d'acteurs et d'outils différents, combinant les catégories d'analyses traditionnelles de l'aide, du commerce et des investissements (Mawdsley, 2012 : 63-65).

La Chine serait aujourd'hui le premier créancier bilatéral du Kenya, avec $12 \%$ de l'encours de la dette externe publique du Kenya (Chaponnière, 2012). On ne dispose cependant pas de chiffres officiels. En effet, les autorités chinoises ne publient pas de ventilation des montants de son aide par pays (Brautigam, 2009: 164 sq.). Selon Mutua Kioko (2012), la part de l'aide chinoise dans le total de l'aide internationale au Kenya serait passée de $0,08 \%$ en 2002 à $13 \%$ en 2005 .

Pour le financement des infrastructures routières au Kenya, la Chine demeurerait néanmoins en troisième position, derrière la Banque mondiale (portefeuille de projets d'environ 900 millions dollars) et la Banque africaine de développement (environ 600 millions dollars), mais devant l'Union européenne (200 millions dollars). Le portefeuille de projets chinois se situerait entre 300 et 500 millions dollars ${ }^{19}$. Cependant, les entreprises

19 Entretien à la Délégation de l'Union européenne, novembre 2012. 
chinoises remportent aussi des appels d'offre de bailleurs internationaux. Les projets réalisés par des entreprises chinoises mais financés par l'aide internationale ne font pas partie de l'aide chinoise, même si les entreprises paraétatiques peuvent bénéficier d'un appui de l'État chinois.

La voie rapide Nairobi-Thika a par exemple été financée en trois tronçons respectivement par l'aide bilatérale chinoise, la Banque mondiale et la Banque africaine, mais réalisée intégralement par des entreprises de BTP chinoises (China Wuyi, Sinohydro et China Shangli). Sur une longueur de $49 \mathrm{~km}$, cette route relie le centre-ville aux quartiers Nord de la capitale jusqu'à la municipalité de Thika dans le district de Kiambu. Première voie rapide du pays, elle a permis de passer d'une simple route à un axe de quatre voies dans chaque sens. Elle a été inaugurée officiellement en novembre 2012 par l'ancien président kenyan Mwai Kibaki après trois années de travaux pour un coût total de 27 milliards KES (environ 310 millions dollars). La route qui relie la Kenya à l'Éthiopie est également construite par trois entreprises chinoises, mais sur financement de la Banque africaine de développement. L'entreprise China Road \& Bridge Corporation (CRBC) aurait construit à elle seule $1000 \mathrm{~km}$ de routes dans le pays (Chaponnière, 2012: 153). CRBC va également construire la ligne de chemin reliant le port de Mombassa à l'Ouganda (Xinhua, 2013).

A l'échelle du continent, les contrats des entreprises chinoises de BTP représentent 40 milliards dollars en 2012, soit une hausse de $45 \%$ depuis 2009. L'Afrique constitue $35 \%$ des marchés de construction chinois à l'étranger (Office d'information du Conseil pour les Affaires d'État). D'après le bureau du conseiller de coopération économique et commerciale de l'Ambassade de Chine, les 25 entreprises chinoises de construction auraient en 2012 un portefeuille de 62 projets dans le pays, pour un montant total de 1,89 milliards dollars (chiffres 2012). Elles réalisent des travaux de construction, de routes, mais aussi de réseaux d'adduction et d'évacuation des eaux, de géothermie. Contrairement aux autres bailleurs intervenant sur le volet infrastructures et développement urbain, les projets chinois sont concentrés sur la dimension opérationnelle et ne contribuent ni à la planification stratégique comme le font par exemple les Japonais à Nairobi ou les Français à Kisumu, ni au renforcement des capacités des institutions locales comme le fait la Banque mondiale. En 2006, l'agence de coopération japonaise (JICA) avait par exemple défini les priorités pour les routes de Nairobi. Les Chinois ont simplement réalisé une étude de faisabilité opérationnelle, suite à la visite de Hu Jintao. 
Les entreprises chinoises contribuent donc à l'amélioration des infrastructures du Kenya, avec l'appui de financements bilatéraux ou multilatéraux : amélioration du réseau routier de Nairobi, renforcement du réseau de distribution électrique, construction et gestion d'une unité de production d'électricité à partir de la géothermie (Olkaria), construction de l'hôpital universitaire de l'Université Kenyatta, routes rurales, construction d'un quai d'amarrage dans le port de Mombassa. En décembre 2012, le Ministère kenyan de l'Énergie a par exemple signé un contrat avec CCC Engineering (CCCE) pour l'amélioration de la transmission d'électricité à haute tension. Le projet est financé par un prêt concessionnel du gouvernement chinois d'un montant d'environ 100 millions dollars. Celui-ci comprend la construction de $392 \mathrm{~km}$ de lignes électriques et de cinq transformateurs, sur une durée de 27 mois (site Internet de CCCE). Les entreprises de construction arrivées dans le pays pour des projets financés par le gouvernement chinois ou des bailleurs internationaux décrochent par la suite des contrats sur une base commerciale pour le secteur privé. La China Wuyi construit par exemple le nouveau siège de la Kenya Commercial Bank (KCB) à Nairobi dans le quartier d'Upper Hill, et construit pour des promoteurs immobiliers chinois.

\section{Échanges commerciaux}

En 2002, le volume commercial des échanges entre la Chine et le Kenya s'élève à 186 millions dollars après avoir dépassé la barre des 100 millions dollars en 1995. Alors que la Chine était le $11^{\text {ème }}$ fournisseur du pays en 2000, elle passe en première place à partir de 2010. Le commerce bilatéral passe à 1,83 milliards dollars en 2010 et 2,4 milliards dollars en 2011, soit une augmentation de $30 \%$ depuis 2000 en base annuelle (Conseiller pour la coopération économique et commerciale de l'Ambassade de Chine à Nairobi). En 2010, le Kenya a exporté pour 40 millions dollars vers la Chine : fruits frais, poisson, ferraille à recycler, minerai, coton, fibre de sisal, cuir, café, thé. La même année, les importations depuis la Chine ont représenté un montant de 1,79 milliards dollars. La Chine exporte au Kenya des équipements électriques, machines outils et équipements de chantiers, des pièces détachées, des matériaux de construction, des véhicules et deuxroues, des produits textiles, des vêtements et chaussures, des produits pharmaceutiques, des équipements agricoles et des biens de consommation (électroménager). La balance commerciale est donc largement positive pour la Chine, ce qui n'est pas le cas à l'échelle du continent, la Chine important un montant de matières premières supérieur à ses exportations vers l'Afrique ${ }^{20}$.

20 La balance commerciale des échanges de la Chine avec l'Afrique est structurellement déficitaire depuis 2004, à l'exception des années 2007 et 2009 (Office d'information du Conseil pour les Affaires d'État). 
A l'échelle du continent, la Chine est devenue le premier partenaire commercial de l'Afrique en 2009 avec un volume d'échange en croissance de 19,3\% par an, soit 198 milliards dollars en 2012 (Office d'information du Conseil pour les Affaires d'État). En 2011, le commerce bilatéral sino-kenyan représentait donc environ 1,5\% des échanges commerciaux entre la Chine et l'Afrique. Les exportations de la Chine vers l'Afrique s'élèvent à 85,3 milliards dollars en 2012 ( $+16,7 \%$ par an), tandis que les importations de la Chine depuis l'Afrique représentent 113,2 milliards dollars en 2012 ( $+21,4 \%$ par an). Ainsi, le Kenya pèse environ $3 \%$ des exportations chinoises, et seulement $0,05 \%$ de ses importations depuis l'Afrique. Cette position particulière du Kenya s'explique par sa relative pauvreté en ressources naturelles qui en fait un fournisseur très modeste pour la Chine. Les Chinois mènent toutefois des explorations dans le sous-sol kenyan. Le Kenya dispose en effet de ressources en fluor, sel, calcaire, cuivre, zinc, aluminium, niobium et thorium.

\section{Investissements}

Les flux d'investissement direct à l'étranger (IDE) chinois au Kenya sont restés faibles avant les années 2000 et ont connu un essor à partir de 2004-2006. En 2002, on dénombrait seulement 17 investissements chinois dans le pays. Une dizaine de nouvelles entreprises chinoises s'est installée en 2003, la plupart dans le secteur des services, dans l'industrie manufacturière, et dans une moindre mesure dans l'exploration minière. Avec un stock de 474 millions dollars en cumulé en 2013, la Chine est devenue la première source d'IDE du Kenya, en augmentation d'environ $30 \%$ par an ${ }^{21}$. Sur l'ensemble du continent, le stock d'IDE chinois s'élevait en 2012 à 14,2 milliards dollars en cumulé. Le Kenya représente donc un peu moins de 2,5\% des investissements chinois en Afrique. Ceux-ci couvrent notamment les secteurs de l'automobile, de l'énergie, des matériaux de construction, de l'immobilier, du tourisme (Bureau du Conseiller pour la coopération économique et commerciale de l'Ambassade de Chine à Nairobi). En 2012, 96 projets d'IDE chinois employaient 6700 Kenyans (Mutua Kioko, 2008 : 7). Les entreprises chinoises opèrent au Kenya en tant qu'entreprises privées bien que la plupart soit détenue partiellement ou en totalité par l'État chinois ${ }^{22}$.

$21 \mathrm{Au}$ Kenya, les IDE sont définis par l'Investment Promotion Act : sont considérés comme investissements directs à l'étranger (IDE) tous les investissements en capitaux étrangers (monnaies étrangères, crédits, droits de propriété), entrepris par une entreprise étrangère dans le but de produire des biens ou services vendus sur le marché intérieur ou exportés.

22 Il s'agit en fait de filiale : une entreprise publique chinoise peut établir des filiales privées à l'étranger. 
On peut citer plusieurs exemples d'investissements productifs : une cimenterie avec unité de production d'électricité (běijīng shübiàndiàn gōngsĭ), qui constitue une première en Afrique de l'Est, une unité d'assemblage de téléviseurs par une entreprise de Qingdao (Aucma, àokēmă), d'une capacité de 100000 téléviseurs par an, une ligne de production de papier toilette et de mouchoirs en papier (kāngdēng gāokējì yǒuxiàn gōngsī), une usine de fabrication de batteries de voitures et de panneaux solaires thermiques par une entreprise du Hunan (xiānghuī gōngyè zhìzào yǒuxiàn gōngsī). En matière de santé, le groupe Beijing Holley-Cotec Pharmaceuticals (huáfāngkētài) a ouvert une plateforme logistique à Nairobi pour desservir l'Afrique centrale et de l'Est (Ambassade de Chine au Kenya).

Ces investissements contribuent à la diversification de l'économie kenyane et sont pourvoyeurs d'emploi, notamment sur les chaines de production. Ils permettent aussi de produire localement des produits traditionnellement importés de l'étranger. Si les capitaux peuvent être rapatriés en Chine, les entreprises sont soumises à la fiscalité locale. Ces investissements diffèrent peu des autres acteurs étrangers, mis à part des coûts de production inférieurs et une meilleure maîtrise des délais. Ils ne sont d'ailleurs pas toujours assortis de succès. Contrairement aux idées reçues, ces entreprises emploient principalement des ouvriers locaux, le Kenya disposant d'une main-d'œuvre relativement qualifiée. Le taux d'emploi local est passé de $82 \%$ en 2003 à $97 \%$ en 2004 (Onjala, 2008 : 13). Sur la question du recours à la main-d'œuvre locale, un cadre d'une entreprise de construction occidentale implantée à Nairobi explique qu'un seul niveau hiérarchique supplémentaire est occupé par un expatrié chinois dans les entreprises chinoises : quand une entreprise occidentale disposera sur un chantier d'un directeur de travaux expatrié et d'un conducteur de travaux local, les deux postes sont occupés par un Chinois dans une entreprise chinoise, le niveau d'en-dessous, chef de chantier, étant occupé par un local. L'émergence de la Chine a été globalement favorable aux pays africains (Kernen, 2007 : 170).

Enfin, l'immobilier résidentiel occupe une place particulière. Une dizaine de promoteurs chinois privés construisent des résidences de logements à Nairobi et dans sa périphérie. Ils sont positionnés sur le marché intermédiaire de l'accession à la propriété pour les classes moyennes. Parfois présentés par les autorités chinoises comme des projets de coopération sino-kenyane (Brautigam, 2009 : 114), ces réalisations ne sont ni de l'aide au développement telle que définie par les critères de l'OCDE, ni des investissements au sens productif du terme : il s'agit de spéculation immobilière, même si certains projets font intervenir des banques publiques chinoises. 
La présence économique chinoise au Kenya est multiforme. Le volet aide au développement contribue à renforcer les acteurs commerciaux, les spéculateurs et les investisseurs.

\section{Le Kenya au cour de la stratégie chinoise en Afrique de l'Est}

La Chine exerce également un rôle plus diffus au Kenya en y développant sa capacité d'attractivité. Nous analyserons dans cette partie les outils de l'influence chinoise et la manière dont la Chine et ses acteurs sont perçus localement.

\section{Les outils du soft power chinois au Kenya}

En matière d'éducation, la Chine s'est engagée dès 1982 à octroyer dix bourses d'études par an pour des étudiants kenyans. Ce nombre a été augmenté avec le renforcement de la coopération sino-africaine dans les années 2000. En 2002, 58 étudiants ont bénéficié d'une bourse pour étudier dans une université chinoise. Le Kenya comptait 306 étudiants en Chine en 2012. Le programme de formations pour les officiels et techniciens kenyans a été renforcé à la fin des années 2000. Le nombre de bénéficiaires est passé de 158 personnes en 2009 à 398 en 2011 (Bureau du Conseiller pour la coopération économique et commerciale de l'Ambassade de Chine à Nairobi). Environ 500 personnes en bénéficieraient désormais (Onjala, 2008 : 35). Lors du quatrième Forum sur la coopération sino-africaine en 2009, la Chine s'était engagée à former 20000 professionnels africains et à augmenter le nombre de bourses d'études de 5500 avant 2012 (Grimm, 2012 : 3). Entre 2010 et 2012, la Chine a permis à 27318 professionnels, fonctionnaires et techniciens, de suivre une formation et d'effectuer un séjour d'études en Chine (Office d'information du Conseil pour les Affaires d'État), soit environ $3 \%$ de Kenyans. Les autorités chinoises ont également octroyé 18743 bourses d'études à des étudiants africains sur la même période, soit un peu moins de $5 \%$ d'étudiants kenyans. Si la qualité des formations dispensées, notamment pour les séjours courts, ne semble pas toujours parfaitement adaptée, ces échanges permettent néanmoins à de nombreux Kenyans de découvrir la Chine et son modèle de développement.

Les échanges culturels passent aussi par la diffusion de la langue chinoise. Le mandarin serait appris par 40 millions de personnes dans le monde. Sous le pilotage du Ministère de l'Education et hébergés dans des établissements académiques à l'étranger, les Instituts Confucius ont pour mission de promouvoir la langue et la culture chinoises dans le monde par l'envoi de professeurs chinois. Ces Instituts incarnent une nouvelle figure du soft power chinois et de la volonté des autorités de développer une influence culturelle 
à l'international (Paradise : 647 sq.). En Afrique, on dénombre au moins 32 instituts Confucius dans 22 pays, dont trois au Kenya. Le premier Institut Confucius d'Afrique a été ouvert en 2005 à l'Université de Nairobi, avant l'ouverture d'un second à l'Université Kenyatta en 2008, puis d'un troisième à l'Université Egerton. Un « cours Confucius » de chinois est également diffusé à la radio dans Nairobi.

Nairobi constitue un point d'entrée en Afrique de l'Est pour les médias officiels chinois en anglais. L'agence de presse Xinhua y a ouvert un bureau dès 1985. La radio chinoise, China Radio International, a commencé à émettre en Afrique depuis Nairobi en février 2006, puis à Mombassa à partir de janvier 2011. La chaîne CCTV Afrique de la télévision nationale chinoise diffuse ses émissions à partir de Nairobi depuis janvier 2012. En décembre 2012, l'édition Afrique du quotidien chinois China Daily a ouvert des bureaux dans la capitale kenyane (Ministère chinois des Affaires étrangères). Il est difficile de connaitre l'audience des médias chinois au Kenya et en Afrique, mais ces derniers témoignent des moyens mis en œuvre par les autorités chinoises pour conforter sa présence dans le domaine de l'information (Francks \& Ribet, 2009 : 129 sq.), au même titre que les pays anglo-saxons ou la France. La Chine fait également des dons de matériel informatique à des journalistes kenyans, comme en 2001 auprès du journal Kenya Times ou en 2004 auprès de l’Union des journalistes kenyans.

En plus de cette influence diffuse pour propager une meilleure connaissance de la Chine en Afrique, le gouvernement chinois exporte son savoir-faire au travers d'un programme d'assistance technique d'E-gouvernement pour le gouvernement kenyan. Le projet vise à créer un système informatique de communication intergouvernementale (Government Unified Messaging System). Ce programme est piloté au niveau de la présidence kenyane. Il va dans le sens des objectifs chinois de diffusion de son propre modèle de développement. «We would like to share our experience », expliquait Han Chunlin, le Conseiller pour la coopération économique et commerciale de l'Ambassade de Chine à Nairobi dans un discours en 2012.

\section{La Chine vue du Kenya}

Le développement chinois est perçu au Kenya comme une réussite même si son application dans le contexte kenyan paraît difficile. Les Chinois jouissent d'une certaine popularité dans la construction des infrastructures (Mutua Kioko, 2012 : 11). La population a le sentiment que le pays progresse en voyant les projets sortir de terre et la 
circulation devenir plus fluide à Nairobi (Daily Nation, 2013). Leur rapidité est louée par les autorités locales : "They are touching the ground very fast », explique M. Stanley, le vice-maire de la municipalité de Mavoko en périphérie Sud de Nairobi . En effet, quand la construction d'une route financée par l'Union européenne nécessite une dizaine d'année entre l'identification du projet et sa livraison, seules quatre années suffisent aux opérateurs chinois. Contrairement aux bailleurs du CAD, la présence des Chinois au contact du terrain est souvent mise en avant pour expliquer la capacité de mener à terme les projets en respectant les délais. Un professeur de l'Université Kenyatta explique : «Development aid from China is helping because they have people on the ground to ensure the follow up » .

Les Chinois font toutefois face à des critiques sur la qualité des réalisations, même si le laxisme des sociétés de supervision peut être également en cause (Chaponnière, 2012 : 154). Plusieurs aspects sont critiqués : les machines ne restent pas sur place à l'issue des travaux ; les salaires de la main-d'œuvre locale seraient insuffisants ; les lois ne seraient pas toujours respectées. Certains interlocuteurs soulignent l'absence de prise en compte d'aspects techniques comme le contrôle de la circulation ou la sécurité routière. Au-delà des réalisations elles-mêmes, la planification semble pâtir du manque d'études préalables. De manière plus générale, l'aide chinoise renforcerait les élites politiques au détriment des populations, affaiblirait les standards sociaux et environnementaux, causerait préjudice à la lutte anticorruption (Mutua Kioko, 2012 : 12). Les Chinois demeurent en concurrence avec d'autres acteurs. Les Japonais qui construisent également des routes à Nairobi pâtissent de critiques sur leur lenteur, mais jouissent d'une image positive en termes de qualité des réalisations.

L'attitude des médias chinois témoigne d'un souci d'image. Plusieurs articles de presse soulignent la manière dont les réalisations des acteurs chinois sont appréciées localement. En 2011 à l'issue de la réhabilitation du barrage de Sasamua dans le centre du pays par l'entreprise chinoise Sinohydro, la Ministre kenyane de l'Eau et de l'irrigation, Charity Ngilu, aurait ainsi loué l'expertise des ingénieurs chinois, en expliquant que les ingénieurs locaux avaient beaucoup à apprendre d'eux. Le Ministre kenyan des routes, Franklin Bett, aurait fait de même en 2010 devant des sociétés locales qui s'étaient plaintes de l'octroi de contrats à des entreprises chinoises. L'ancien Premier Ministre Raila Odinga, qui est ingénieur de formation, aurait lui-même encouragé les sociétés locales à améliorer leur efficacité face aux entreprises chinoises (People's Daily, 2011). Les médias chinois font également appel aux propos d'experts locaux pour donner une image positive de la Chine : 
Edward Oyugi, professeur à l'Université de Nairobi a ainsi souligné que l'aide chinoise est fondamentale pour le développement du Kenya et de l'Afrique dans la mesure où elle cible les infrastructures (Xinhua, 2010). La Chine se pose ainsi en partenaire incontournable du développement africain.

\section{Conclusion}

Les relations entre la Chine et le Kenya ont pris une importance majeure dans les années 2000, même si la structure des relations ne diffère pas fondamentalement des autres partenaires du Kenya. Vue de Chine, l'Afrique n'est pas seulement un espace pourvoyeur de ressources naturelles : le continent constitue une terre d'occasions économiques, où la Chine ambitionne d'accroitre son influence. Le Kenya en est une singulière illustration. Le pays constitue un partenaire stratégique par sa localisation, sa relative stabilité politique et son poids économique dans la région.

Contrairement à ce que pourrait laisser croire l'anecdote du mariage d'une ancienne Miss Kenya avec le fils d'un riche homme d'affaire du Sichuan en 2010, les différences culturelles et de contextes de développement demeurent. En dépit de son pouvoir d'attraction sur les autorités kenyanes, le modèle de développement chinois ne saurait être applicable au cas du Kenya. La Chine n'en demeure pas moins un partenaire désormais indispensable au développement du pays. Sa présence témoigne de la capacité d'insertion des différents acteurs chinois dans des secteurs variés de l'économie kenyane, en mobilisant et combinant les outils d'aide au développement, les investissements et les échanges commerciaux.

À l'heure où le Président Uhuru Kenyatta et son vice-président William Ruto sont critiqués par les États-Unis et la Grande-Bretagne suite aux accusations du Tribunal pénal international (TPI) liés aux violences postélectorales de 2007-2008, le Kenya pourrait aspirer à travailler plus étroitement encore avec la Chine, dont les autorités se refusent à toute ingérence, et tout commentaire, dans les affaires intérieures des États. 
Les secteurs de la présence chinoise au Kenya

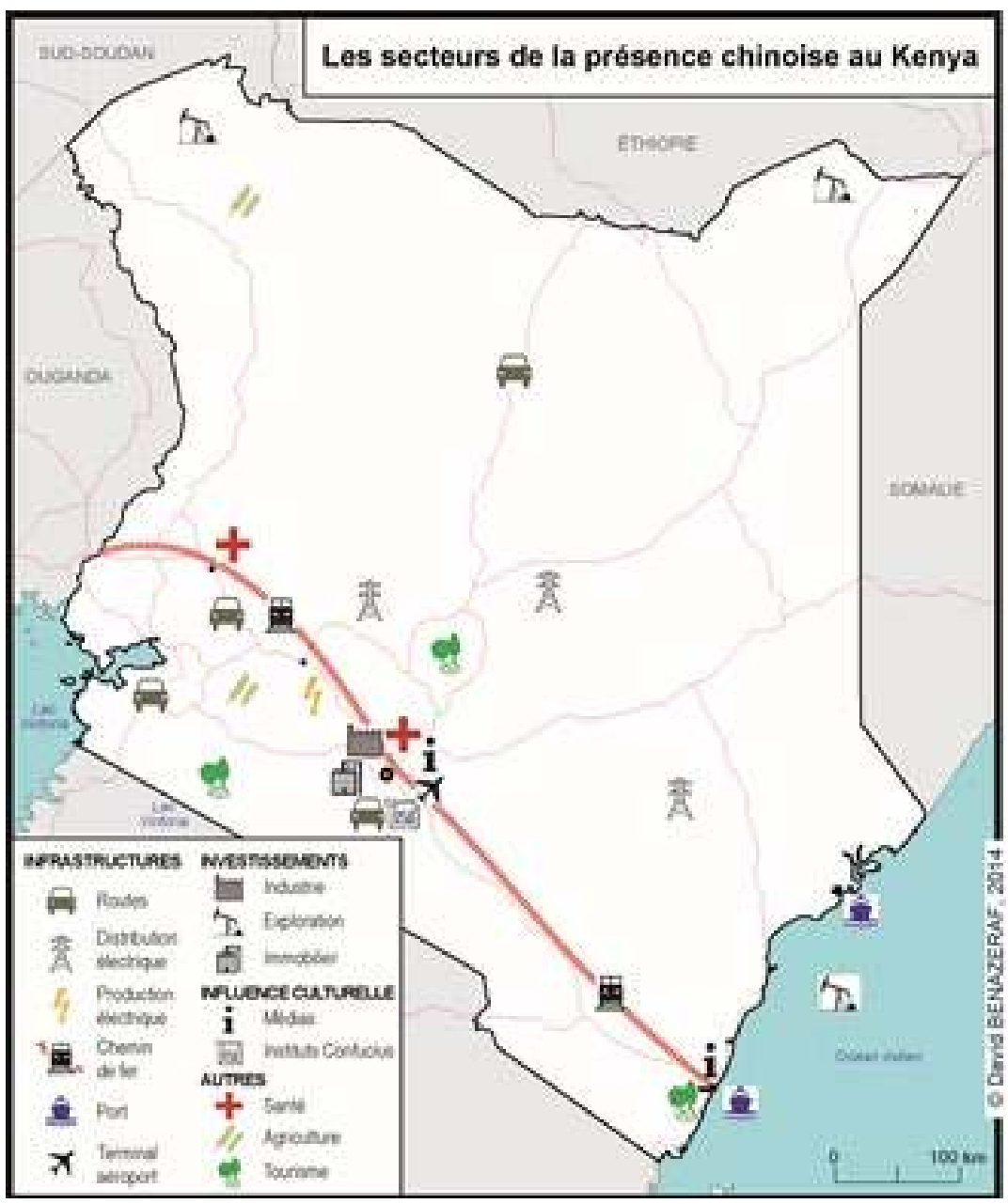




\section{Bibliographie}

Africa Research Institute. «Interview, Ambassador Zhong Jianhua China’s Special Representative on African Affairs » (10 mai 2013).

Alden, Chris, Daniel Large, Ricardo Soares de Oliveira. China Rreturns to Africa: a Rising Power and a Continent Embrace. London: Hurst, 2008.

Amelot, Laurent. « Le Kenya, futur pivot de la politique énergétique chinoise au SudSoudan et au-delà ? ». Outre-Terre 30 (2011) : 187-195.

Bezbakh, Pierre. « Zheng He, 'l'amiral des mers de l'Ouest'». Le Monde (08/11/2011)

Brautigam, Deborah. The Dragon's Gift: the Real Story of China in Africa. Oxford: Oxford University press, 2009.

Cabestan, Jean-Pierre. « La Chine et l'Éthiopie : entre affinités autoritaires et coopération économique ». Perspectives chinoises 4 (2012) : 57-68.

Chai, Zhengxing, Shengyue Hao \& Lianwei Guo. "Internationalization Strategies for China's Construction Enterprises", Beijing: School of economics and management, Beijing Jiaotong University, 2010 [consulté le 17/06/2013] URL: http://www.seiofbluemountain.com/upload/product/201001/1263175505g5chqvft.pdf .

Chaponnière, Jean-Raphaël \& Jean-Jacques Gabas (dir.). Le temps de la Chine en Afrique: enjeux et réalités au sud du Sahara. Paris : GEMDEV, Karthala, 2012.

Chaponnière, Jean-Raphaël. « Un demi-siècle de relations Chine-Afrique, Evolution des analyses ». Afrique contemporaine, 228 (2008) : 35-48.

Charnoz, Olivier \& Jean-Michel Severino. L'aide publique au développement. Paris : La Découverte, 2007.

Chine, Office d'Information du Conseil des Affaires d'État. Zhongguo yu feizhou de jingmaohezuo de pishu [Livre blanc de la Coopération économique et commerciale entre la Chine et l'Afrique]. Pékin, 2013.

Clapham, Cristopher. « Fitting China in ». Brenthurst discussion paper (août 2006).

Decherf, Dominique. Couleurs, Mémoires d'un ambassadeur de France en Afrique. Paris: Pascal Galodé Éditeurs, 2012.

Francks Suzanne \& Kate Ribet. « China in Africa. What Does it Mean for the Media? ». 
Global Media and Communication 5, no. 1 (2009): 129-136.

Grimm, Sven. "The Forum on China-Africa Cooperation (FOCAC), Political Rationale and Functioning". Stellenbosch University: Centre for Chinese Studies, Policy Briefing (mai 2012).

Kernen, Antoine. « Les stratégies chinoises en Afrique : du pétrole aux bassines en plastique ». Politique africaine 105 (2007): 163-180

Li, Anshan, 中国对非政策的调适与转变 (zhōngguó duì fēi zhèngcè de tiáoshì yŭ zhuănbiàn, “On the Adjustment and Transformation of China's Africa Policy”). 《西亚非 洲》 (xīyă fẽizhōu, West Asia and Africa) 8 (2006): 11-20.

Monson, Jamie, RUPP Stephanie, "Introduction: Africa and China: New Engagements, New Research". African Studies Review 56, no. 1 (2013): 21-44

Mutua Kioko, Patrick. «A Study on Chinese Economic relations with Africa: Case Study, Kenya ». Prime Journal of Business Administration and Management 2, no. 3 (2012): 485-496.

Nye, Joseph. Soft Power: The Means to Success in World Politics. New York: Public Affairs, 2004.

Onjala, Joseph. A Scoping Study on China-Africa Economic Relations: the Case of Kenya. University of Nairobi, Institute for Development Studies (mai 2008).

Pairault, Thierry, «L'investissement direct chinois en Afrique ». Outre-Terre 30 (2011) : 89-114.

Paradise, James F. « China and International Harmony: The Role of Confucius Institutes in Bolstering Beijing's Soft Power ». Asian Survey 49, no. 4 (2009): 647-669.

Tan-Mullins, May, Giles Mohan \& Marcus Power. « Redefinig 'aid' in the China-Africa context ». Development and Change 45, no. 5 (2010): 857-881.

Taylor, Ian. The Forum on China-Africa Cooperation. New-York: Routledge, 2011.

Véron, Jean-Bernard. «Éditorial ». Afrique contemporaine 228 (2008) : 5-9.

Will, Rachel. « China's Stadium Diplomacy ». World Policy Journal 29, no. 2 (2012): 36-43. 
« Chinese bank-financed housing project kicked off in Kenya ». Xinhua (31/05/2008) [consulté le 08/08/2012] URL: http://news.xinhuanet.com/english/2008-05/31/ content_8287705.htm

« Le Kenya et Singapour s'engagent à renforcer leurs relations ». Xinhua (22/01/2010) [consulté le 08/01/2014] URL : http://french.peopledaily.com.cn/International/6875791. html

« Kenyan minister praises Chinese workmanship ». People's Daily (28/04/2011)

«Kenya : projet de la super autoroute de Thika ». cntv.cn (19/07/2012) [consulté le 17/05/2013], URL : http://french.mofcom.gov.cn/article/zt_fslcsa/ lanmufc/201207/20120708242192.shtml

«Thika superhighway becomes model road in rainy season ». Daily Nation (08/04/2013) [consulté le 21/05/2013] URL : http://www.nation.co.ke/News/Thika-superhighwaybecomes-model-road-in-rainy-season/-/1056/1742508/-/13e2hxz/-/index.html « Roads and energy main beneficiaries of Chinese aid to Kenya ». Africa Review (30/04/2013) [consulté le 08/07/2013] URL : http://www.africareview.com/ Business---Finance/Roads-and-energy-main-beneficiaries-of-Chinese-Kenya-aid//979184/1762638/-/4eqya2z/-/index.html

« Kenya signs infrastructure, energy deals worth $\$ 5$ billion with China ». Reuters (19/08/2013) [consulté le 21/08/2013] URL : http://www.reuters.com/article/2013/08/19/ us-kenya-china-idUSBRE97I0RV20130819

« Kenya official says Chinese funding revives mega-infrastructure projects ». Xinhua (27/11/2013) [consulté le 07/01/2014] URL : http://www.chinadaily.com.cn/ xinhua/2013-11-27/content_10684392.html

Site du Conseiller de coopération économique et commerciale de l'Ambassade de Chine au Kenya (Mofcom) [consulté le 28/10/2013] URL : http://ke2.mofcom.gov.cn Site du Ministère chinois des affaires étrangères (Waijiaobu) [consulté le 28/10/2013] URL : http://www.fmprc.gov.cn/mfa_chn/gjhdq_603914/gj_603916/ fz_605026/1206_605656/

Site Engineering News record [consulté le 28/10/2013] URL: http://enr.construction. com/toplists/Top-International-Contractors/001-100.aspi

Site de l'entreprise CCC Engineering (version chinoise) : http://www.ccceng.cn/news detail.aspx?NewsCateID=89\&NewsID=89\&CateID=13 [consulté le 07/01/2014] 